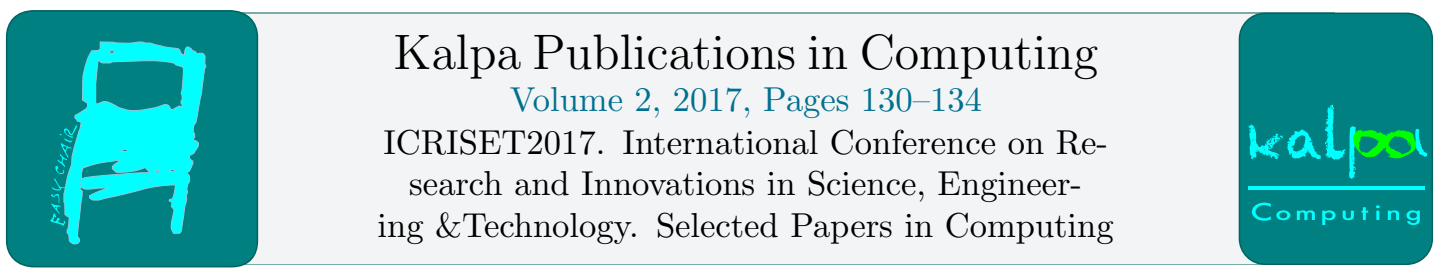

\title{
Weakly analytic sets for the Cartesian product of function algebra
}

\author{
Himali S. Mehta ${ }^{1}$, Rekha D. Mehta ${ }^{2}$, Aakar N. Roghelia ${ }^{3}$ \\ ${ }^{1,2}$ Department of Mathematics, Sardar Patel University, Vallabh Vidyanagar, India \\ ${ }^{3}$ Department of Mathematics, BVM Engineering College, Vallabh Vidyanagar, India \\ ${ }^{1}$ hs mehta@spuvvn.edu, ${ }^{2}$ vvnspulyahoo.co.in, \\ ${ }^{3}$ aakar.roghelia@bvmengineering.ac.in
}

\begin{abstract}
Weakly analytic sets for function algebra is studied by Arenson in (Arenson). Here, we study the concept of weakly analytic sets for Cartesian product of function algebras. We express the weakly analytic sets for Cartesian product of function algebra in terms of that for factor algebras.
\end{abstract}

\section{Weakly analytic sets}

The concept of weakly analytic sets for a function algebra was introduced by Arenson (Arenson). The weakly analytic property is stronger than antisymmetry. The decomposition given by maximal antisymmetric sets for the Cartesian product is obtained in (Mehta and Mehta). The weakly analytic sets induces a decomposition which is finer than the Bishop decomposition. Here, we have obtained weakly analytic sets for the Cartesian product of function algebras in terms of factor algebras.

Let $X$ be a compact, Hausdorff space and $C(X)$ be the Banach algebra of complex valued continuous functions on $X$. A complex function algebra is a closed subalgebra of $C(X)$, which contains constants and separates the points of $X$.

\section{Examples 1.1}

1. Trivially, $C(X)$, where $X$ is a compact, Hausdorff space is a function algebra on $X$.

2. Let $D=\{z \in \mathbb{C}:|z| \leq 1\}$. Then, $A(D)$ the set of all functions continuous on $D$ and analytic in the interior of $D$ is a function algebra on $D$, known as the Disc algebra.

3. Let $D_{1}=\{z \in \mathbb{C}:|z| \leq 1\}, D_{2}=\{z \in \mathbb{C}:|z-2| \leq 1\}$ and $D=D_{1} \cup D_{2}$. Also, let 
$A=\left\{f \in C(D): f\right.$ isanalyticin $\left.D^{o}\right\}$, where $D^{o}$ denote interior of $D$. Then, $A$ is a function algebra on $D$.

We start with some basic concepts related to the Cartesian product of function algebras.

Let $X$ and $Y$ are two compact, Hausdorff topological spaces with topologies $\tau_{X}$ and $\tau_{Y}$ respectively. Then, the disjoint union $X \cup Y$ is a compact, Hausdorff topological space with the sum topology $\tau$ defined as $\tau=\left\{G \subset X \cup Y: G \cap X \in \tau_{X}, G \cap Y \in \tau_{Y}\right\}$ (Shah). We denote this topological space by $X+Y$. Also, the mapping $T: C(X) \times C(Y) \rightarrow C(X+Y)$ defined by $T((f, g))=h$ where

$$
h(w)= \begin{cases}f(w), & \text { if } w \in X \\ g(w), & \text { if } w \in Y\end{cases}
$$

is an algebra isomorphism from $C(X) \times C(Y)$ on to $C(X+Y)$, where $C(X) \times C(Y)$ is Cartesian product of $C(X)$ and $C(Y)$ defined as $C(X) \times C(Y)=\{(f, g): f \in C(X), g \in C(Y)\}$.

If $A$ is a function algebra on $X, B$ is a function algebra on $Y$, then $A \times B$ is a function algebra on $X+Y$ with maximum norm defined as $\|h\|=\max \{\|f\|,\|g\|\}$ for $h=(f, g) \in A \times B$ (Patel).

Remark 1.2 For a closed subset $E \subset X+Y$, we can easily verify that $(A \times B)_{E}=A_{(E \cap X)} \times B_{(E \cap Y)}$, where $A_{F}$ denotes the uniform closure of the restriction algebra $A_{\mid F}$ in $C(F)$ for a closed subset $F$ of $X$. Also, if $E \subset X$ (respectively $E \subset Y)$ then $(A \times B)_{E} \cong A_{E}\left(\right.$ respectively $\left.(A \times B)_{E} \cong B_{E}\right)$.

We need the definition of peak sets and weak peak sets for a function algebra which are required for our main result. Now onwards, $A$ and $B$ will denote function algebras on $X$ and $Y$ respectively.

Definition 1.3 A nonempty subset $S$ of $X$ is called a peak set for $A$ if here exists $f \in A$ such that $f_{\mid S}=1$ and $|f(y)|<1$ for all $y \in X \backslash S$. The function $f$ is called a peaking function on $S$. The set $S$ is called a weak peak set for $A$ if it is an intersection of some collection of peak sets.

For peak sets we know that

1. If $S($ respectively $T)$ is a peak set for $A(\operatorname{respectively} B)$, then $S($ respectively $T)$ is a peak set for $A \times B$. Hence, $S \cup T$ is a peak set for $A \times B$.

2. If $S$ is a peak set for $A \times B$, then $S \cap X$ and $S \cap Y$ are peak sets for $A$ and $B$ respectively.

Above results also remain valid if we replace "peak set" by "weak peak set".

Next, we recall the definition of weakly analytic sets.

Definition 1.4 (Arenson) A closed subset $E$ of $X$ is called a weakly analytic set for $A$, if every peak set $S$ for $A_{E}$, either coincides with $E$ or it is nowhere dense in $E$.

Equivalently, $E$ is a weakly analytic set for $A$ if $E=S \cup T$, where $S$ is a peak set for $A_{E}$ and $T$ is a closed set in $E$, then either $S=E$ or $T=E$.

$A$ is called weakly analytic algebra if $X$ is a weakly analytic set for $A$. Following result is known for weakly analytic sets. 
Proposition 1.5 (Arenson)

1. Each weakly analytic set for $A$ is contained in a maximal weakly analytic set for $A$.

2. Each weakly analytic set is antisymmetric.

We denote the set of all maximal weakly analytic sets for $A$ by $\mathcal{W}(A)$. Now, we will determine weakly analytic sets for some function algebras.

Example 1.6 The function algebra $A(D)$ is a weakly analytic algebra can be checked easily.

Example 1.7 Let $D=D_{1} \cup D_{2}$, where $D_{1}=\{z \in \mathcal{C}:|z-1| \leq 1\}$ and $D_{2}=\{z \in \mathcal{C}:|z+1| \leq 1\}$. Let $A=A(D)$, the set of all functinons in $C(D)$ which are analytic in the interior of $D$. Then $A$ is a function algebra on $D$ [3]. It can be checked that $\mathcal{W}(A)=\left\{D_{1}, D_{2}\right\}$.

Example 1.8 Let $D$ be the set as in the Example 1.7. Let $X$ be the quotient space of $D$ by identifying the points -1 and $0 ; 1$ and 2 and $q: D \rightarrow X$ be the quotient map. Also, $A=\{f \in C(X): f o q$ is analytic in the interior of $D\}$. Then, $A$ is a function algebra on $X$. $\mathcal{W}(A)=\left\{q\left(D_{1}\right), q\left(D_{2}\right)\right\}$. We can see that $q\left(D_{1}\right)$ is a peak set for $A$ but $q\left(D_{2}\right)$ is not a peak set for A.

\section{Remarks 1.9}

1. Unlike maximal antisymmetric sets, maximal weakly analytic sets for $A$ need not be disjoint as we will see in Example 1.7, $D_{1}$ and $D_{2}$ are maximal weakly weakly sets for $A$, but $D_{1} \cap D_{2} \neq \phi$.

2. Also, maximal weakly analytic sets need not be weak peak sets as we can see in Example 1.8 .

\section{Weakly analytic sets for Cartesian product of function algebra}

Now, we prove the relation between weakly analytic sets for $A \times B$ and that for $A$ and $B$.

Theorem 2.1 If $E \subset X($ respectively $E \subset Y)$ is a weakly analytic set for $A($ respectively $B)$, then $E$ is a weakly analytic set for $A \times B$.

Proof. Let $E$ be a weakly analytic set for $A$ and $S$ be a peak set for $(A \times B)_{E}$. Then $S$ is a peak set for $A_{E}$. So, $S=E$ or $S$ is nowhere dense in $E$. So, $E$ is a weakly analytic set for $A \times B$.

Similarly, if $E \subset Y$ is weakly analytic set for $B$, then $E$ is a weakly analytic set for $A \times B$.

Theorem 2.2 If $E$ is a weakly analytic set for $A \times B$, then either $E \subset X$ or $E \subset Y$. Further, if $E \subset X$ (respectively $E \subset Y$ ), then $E$ is a weakly analytic set for $A$ (respectively $B)$.

Proof. Let $E$ be a weakly analytic set for $A \times B$. Now, $E=(E \cap X) \cup(E \cap Y)$ and $E \cap X$ is a peak set for $(A \times B)_{E}$ as $X$ is a peak set for $A \times B$ and $E$ is a closed set in $X+Y$ (Browder). Also, $E \cap Y$ is a closed subset of $E$. So, $E=E \cap X$ or $E=E \cap Y$ as $E$ is a weakly analytic set for $A \times B$. So, $E \subset X$ or $E \subset Y$. 
Now suppose, $E \subset X$, and $S$ be a peak set for $A_{E}$. Then, $S$ is a peak sets for $(A \times B)_{E}$. So, $E=S$ or $S$ is nowhere dense in $E$. Hence, $E$ is a weakly analytic set for $A$.

Similarly, if $E \subset Y$ then $E$ is a weakly analytic set for $B$.

Theorem 2.3 $\mathcal{W}(A \times B)=\{E: E \in \mathcal{W}(A)\} \cup\{F: F \in \mathcal{W}(B)\}$

Proof. Let $E \in \mathcal{W}(A \times B)$. Then, $E \subset X$ or $E \subset Y$ by Theorem 2.2. Suppose, $E \subset X$, then by Theorem $2.2 E$ is a weakly analytic set for $A$. If $E \notin \mathcal{W}(A)$, then there exists $F \subset X$ which is weakly analytic set for $A$ and $E \subsetneq F$. But, then $F$ is a weakly analytic set for $A \times B$ by Theorem 2.1, which contradicts the maximality of $E$ in $A \times B$. So, $E \in \mathcal{W}(A)$.

Similarly, if $E \subset Y$, then $E \in \mathcal{W}(B)$.

Conversely, if $E \in \mathcal{W}(A)$ or $E \in \mathcal{W}(B)$ then $E \in \mathcal{W}(A \times B)$ by similar arguments.

\section{Examples}

\section{Example 3.1}

Let $X=\{z \in \mathbb{C}:|z-3| \leq 1\}$ and $A=\{f \in C(X): f$ is analytic in the interior of $X\}$. Also, let $Y=\{z \in \mathbb{C}:|z+3| \leq 1\}$ and $B=\{f \in C(Y): f$ is analytic in the interior of $Y\}$.

Then, $\mathcal{W}(A)=X, \mathcal{W}(B)=Y$ and $\mathcal{W}(A \times B)=\{X, Y\}$. Here, $A$ and $B$ are weakly analytic but $A \times B$ is not weakly analytic.

\section{Example 3.2}

Let $X=D_{1} \cup D_{2}$ where $D_{1}=\{z \in \mathbb{C}:|z-2| \leq 1\}, \quad D_{2}=\{z \in \mathbb{C}:|z-4| \leq 1\}$, Let $X^{\prime}$ be the quotient space obtained from $X$ by identifying the points 2 and $3 ; 4$ and 5 and $q_{1}: X \rightarrow X^{\prime}$ be the quotient map. Let $Y=\left\{z \in \mathbb{C}:|z| \leq \frac{1}{2}\right\}$.

Also, let $A=\left\{f \in C\left(X^{\prime}\right): f o q_{1}\right.$ is analytic in the interior of $\left.X\right\}$

and $B=\{f \in C(Y): f$ is analytic in the interior of $Y\}$.

Then, $\mathcal{W}(A)=\left\{q_{1}\left(D_{1}\right), q_{1}\left(D_{2}\right)\right\}, \mathcal{W}(B)=Y$. Hence, $A$ is not weakly analytic but $B$ is weakly analytic. Also, $\mathcal{P}(A \times B)=\left\{q_{1}\left(D_{1}\right), q_{1}\left(D_{2}\right), Y\right\}$. So, $A \times B$ is not weakly analytic.

Example 3.3 Let $X=D_{1} \cup D_{2}$ and $Y=D_{3} \cup D_{4}$, where $D_{1}=\{z \in \mathbb{C}:|z-2| \leq 1\}$, $D_{2}=\{z \in \mathbb{C}:|z-4| \leq 1\}, D_{3}=\{z \in \mathbb{C}:|z+2| \leq 1\}$ and $D_{4}=\{z \in \mathbb{C}:|z+4| \leq 1\}$.

$A=\{f \in C(X): f$ is analytic in the interior of $X\}$ and

$B=\{f \in C(Y): f$ is analytic in the interior of $Y\}$. Then, $\mathcal{W}(A)=\left\{D_{1}, D_{2}\right\}, \mathcal{W}(B)=\left\{D_{3}, D_{4}\right\}$ and $\mathcal{W}(A \times B)=\left\{D_{1}, D_{2}, D_{3}, D_{4}\right\}$.

Remark 3.4 In Examples 3.1, 3.2 and 3.3 we can observe that $A \times B$ is never weakly analytic. 


\section{References}

[1] Arenson, E. L. "Certain Properties of algebras of continuous functions." Soviet Math. Dokl. Vol. 7 (1966): 1522-1523.

[2] Browder, A. Introduction to function algebras. New York: W. A. Benjamin, Inc., 1969.

[3] Ellis, A. J. "Weakly prime compact convex set and uniform algebras." Math. Proc. Camb. Phill. Soc. Vol. 81 (1977): 225-232.

[4] Mehta H. S., Mehta R. D. and Patel D. R. "Essential set and antisymmetric sets for Cartesian product of function algebras." Mathematics Today, Vol. 29 (2013): 25-30.

[5] Patel, D. R. A study of product of Banach algebras and function algebras. Ph. D. Thesis. Sardar Patel University, March, 2016.

[6] Shah, N. H. Cartesian product of Banach algebras. M. Phil., Dissertation. Sardar Patel University, September, 2007. 